\title{
Edge effect causes apparent fractal correlation dimension of uniform spatial raindrop distribution
}

\author{
R. Uijlenhoet ${ }^{1}$, J. M. Porrà ${ }^{2}$, D. Sempere Torres ${ }^{3}$, and J.-D. Creutin ${ }^{4}$ \\ ${ }^{1}$ Chair of Hydrology and Quantitative Water Management, Department of Environmental Sciences, Wageningen University, \\ Wageningen, The Netherlands \\ ${ }^{2}$ VENCA, Vilanova i la Geltrú, Spain \\ ${ }^{3}$ Grup de Recerca Aplicada en Hidrometeorologia, Universitat Politècnica de Catalunya, Barcelona, Spain \\ ${ }^{4}$ Laboratoire d'étude des Transferts en Hydrologie et Environnement, Grenoble, France
}

Received: 28 November 2007 - Revised: 31 March 2009 - Accepted: 31 March 2009 - Published: 9 April 2009

\begin{abstract}
Lovejoy and Schertzer (1990a) presented a statistical analysis of blotting paper observations of the (twodimensional) spatial distribution of raindrop stains. They found empirical evidence for the fractal scaling behavior of raindrops in space, with potentially far-reaching implications for rainfall microphysics and radar meteorology. In particular, the fractal correlation dimensions determined from their blotting paper observations led them to conclude that "drops are (hierarchically) clustered" and that "inhomogeneity in rain is likely to extend down to millimeter scales". Confirming previously reported Monte Carlo simulations, we demonstrate analytically that the claims based on this analysis need to be reconsidered, as fractal correlation dimensions similar to the ones reported (i.e. smaller than the value of two expected for uniformly distributed raindrops) can result from instrumental artifacts (edge effects) in otherwise homogeneous Poissonian rainfall. Hence, the results of the blotting paper experiment are not statistically significant enough to reject the Poisson homogeneity hypothesis in favor of a fractal description of the discrete nature of rainfall. Our analysis is based on an analytical expression for the expected overlap area between a circle and a square, when the circle center is randomly (uniformly) distributed inside the square. The derived expression $\left(\pi r^{2}-8 r^{3} / 3+r^{4} / 2\right.$, where $r$ denotes the ratio between the circle radius and the side of the square) can be used as a reference curve against which to test the statistical significance of fractal correlation dimensions determined from spatial point patterns, such as those of raindrops and rainfall cells.
\end{abstract}

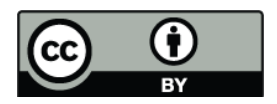

Correspondence to: R. Uijlenhoet (remko.uijlenhoet@wur.nl)

\section{Introduction}

Detailed knowledge of the microstructure of precipitation is important from both a fundamental and an applied point of view (e.g., Uijlenhoet and Sempere Torres, 2006). The spatial and temporal distributions of precipitation particles (hydrometeors) in the atmosphere: (1) determine the manner in which the concept of a particle size distribution should be interpreted (e.g., Porrà et al., 1998; Jameson and Kostinski, 2001a; Kostinski et al., 2006); (2) have important implications for the microphysical processes involving interactions between hydrometeors, such as the efficiency of the collision-coalescence process in producing rainfall (e.g., Pruppacher and Klett, 1978; Rogers and Yau, 1996; Kostinski and Shaw, 2005); (3) strongly influence the sampling characteristics of both in situ and remote sensing instruments used for measuring the microstructure of precipitation, such as disdrometers (e.g., Smith et al., 1993; Uijlenhoet et al., 2006) and vertically pointing Doppler radars.

With regard to raindrops (but the same holds for cloud droplets), the classical hypothesis is that they behave according to Poisson statistics, i.e. that they are as homogeneously distributed in space and time as randomness allows. This hypothesis forms the basis of the sampling theory of in situ rainfall observations (e.g., Cornford, 1967; Joss and Waldvogel, 1969; Gertzman and Atlas, 1977) and can be considered one of the cornerstones of the physical theory of precipitationinduced pulse-to-pulse echo fluctuations in weather radar observations (Marshall and Hitschfeld, 1953; Wallace, 1953). Although traces of empirical evidence for the Poisson homogeneity hypothesis during rare periods of exceptionally stationary rainfall have occasionally been reported in the literature (e.g., Kostinski and Jameson, 1997; Uijlenhoet et al.,

Published by Copernicus Publications on behalf of the European Geosciences Union and the American Geophysical Union. 
1999; Larsen et al., 2005), it now becomes more and more clear that rainfall exhibits pronounced spatial and temporal drop clustering. Since the homogeneous Poisson process (which per definition exhibits a constant mean intensity) is not able to cope with these types of clustering, more versatile descriptions of raindrop statistics are needed.

There exist different approaches to tackling this problem. One consists of generalizing the restrictive homogeneous Poisson process to a Poisson process with a randomly varying mean, that is a so-called doubly stochastic Poisson process or Cox process (e.g., Cox and Isham, 1980). This type of approach was pioneered by Sasyo (1965) and has later been applied by Smith (1993). Kostinski and Jameson (1997) and Larsen et al. (2005) have proposed alternative non-Poissonian yet statistically homogeneous descriptions of rain. Another approach is the (multi-)fractal description of rain, based on models which have originally been used to describe turbulence. Since rainfall is intimately related to the (turbulent) wind field in the atmosphere, it seems natural to employ the same type of models for describing rain fields (e.g., Lovejoy and Schertzer, 1990b). There have also been recent approaches to describing inhomogeneous rainfall via non-fractal methods (e.g., Jameson, 2007). Finally, confirming the earlier hypothesis of Fabry (1996), Lovejoy and Schertzer $(2006,2008)$ have recently shown how the multifractal and Poisson descriptions of rain can be reconciled.

Probably the first application of fractal geometry to describe the discrete nature of rainfall was the statistical (correlation dimension) analysis of the (two-dimensional) spatial distributions of raindrop stains on pieces of blotting paper reported by Lovejoy and Schertzer (1990a, LS hereafter). Later, Zawadzki (1995), Lavergnat and Golé (1998) presented fractal analyses of the (one-dimensional) temporal distribution of raindrop arrivals at the ground (such analyses were recently challenged by Larsen et al., 2005). Desaulniers-Soucy et al. (2001), Lovejoy et al. (2003) and Lilley et al. (2006) reported on multi-fractal analyses of (three-dimensional) spatial distributions of raindrops measured using stereo-photography. Here we concentrate on the blotting paper experiment of LS, which has lead to a lively debate in the scientific literature concerning the statistical significance of the supposed fractal nature of the microstructure of rain (Jameson and Kostinski, 1998; Gabella et al., 2001; Jameson and Kostinski, 2001b; Gabella and Perona, 2001).

\section{Blotting paper experiment and fractal analysis}

The experiment of LS consisted of exposing three $128 \times 128 \mathrm{~cm}^{2}$ pieces of chemically treated blotting paper during exposure times of approximately $1 \mathrm{~s}-$ to "a moderately heavy stratiform rain" at McGill University in Montreal, Canada. One of the blotting papers was digitized, the other two were manually analyzed. The experiment re- sembled the famous experiments carried out more than four decades earlier by Marshall and Palmer (1948) at the same university. The statistical analysis of the digitized blotting paper consisted of drawing concentric circles with increasing radii around the centers of each of a total of 452 raindrop stains. The radii of the circles were increased from a few $\mathrm{mm}$ (i.e. of the order of the size of the stains) to more than $1.5 \mathrm{~m}$ (i.e. largely exceeding the size of the blotting paper) in 10 equal logarithmically spaced steps per decade. For each value of the radius, the numbers of drop stains falling inside each of the 452 circles were averaged. In this manner, LS obtained an average number of drop stains as a function of the circle radius. This empirical function was subsequently plotted on log-log paper (see their Fig. 2).

Apart from a "fall-off" both at small radii (due to the finite number of drop stains in the sample) and at large radii (due to the finite size of the blotting paper), the function was found to be reasonably well described by a straight line for "the part of the graph [...] that was relatively unaffected by the [...] fall-off". This part of the graph was reported to be the range between $2 \mathrm{~mm}$ and $40 \mathrm{~cm}$, although from Fig. 2 of LS it appears that the straight line was actually fitted to the data points within the range between $3 \mathrm{~mm}$ and $63 \mathrm{~cm}$. The obtained slope of the straight line, interpreted by LS as a fractal correlation dimension (e.g., Grassberger and Procaccia, 1983a,b; Theiler, 1987), was found to be 1.83 , with reported values of 1.79 and 1.93 for the two other, manually analyzed, samples. These values are significantly smaller than a slope of 2 that would be expected for uniformly distributed raindrop stains resulting from a homogeneous Poisson process.

LS interpreted this result as "evidence that rainfall is scaling over this range" and concluded that "drops are (hierarchically) clustered over the range". They also discussed the profound implications of this clustering for radar remote sensing of rainfall. For instance, there would no longer be a simple proportionality between the expected number of raindrops in a radar sample volume and the size of that volume. In addition, due to increased coherent scattering, it would strongly affect the statistics of the sample-to-sample radar echo fluctuations as well. Hence, it would essentially be necessary to revise the theory of weather radar (Marshall and Hitschfeld, 1953; Wallace, 1953).

The conclusions of LS have recently been put into perspective by several authors. Both Jameson and Kostinski (1998) and Gabella et al. (2001) have presented results of Monte Carlo simulation experiments intended to mimic LS's blotting paper analysis (see Jameson and Kostinski, 2001b; Gabella and Perona, 2001, for subsequent discussions). Even though these numerical simulation experiments were based on 452 uniformly distributed raindrops, consistent with the Poisson homogeneity hypothesis, both studies reported (nearly) the same fractal correlation dimensions as those found earlier in real rain by LS. These simulation results indicate that the fractal correlation dimension reported by LS may have been merely a sampling artefact, an edge 


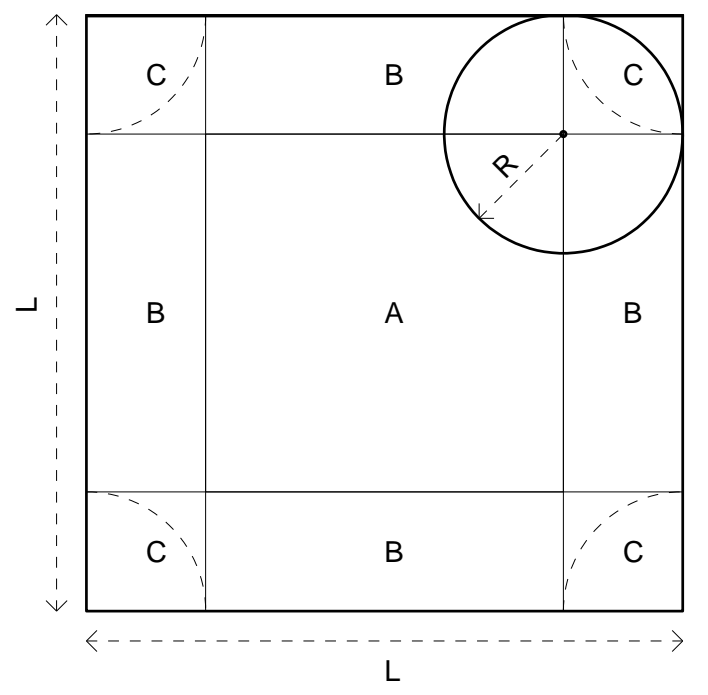

Fig. 1. Schematic representation of a $L \times L$ piece of chemically treated blotting paper (bold square) with a raindrop stain (black dot) and a circle with radius $R(0 \leq R \leq L / 2)$ around the center of the stain (bold circle). The surface of the blotting paper can be divided into three separate regions (as indicated by the thin lines) according as to whether the circle surrounding a raindrop center will fall entirely inside (region A) or partly outside the boundary of the blotting paper (regions B and C).

effect to be more precise. That this may indeed be a valid explanation can be demonstrated analytically, as will be shown in the next section.

\section{Analytical solution to the edge effect}

Figure 1 provides a schematic representation of the blotting papers employed by LS in their statistical analysis. For homogeneously distributed drops (obeying Poisson statistics), the expected number of raindrop stains falling inside a circle of a given radius would be the product of (1) the expected spatial stain density (in this case $452 / 1.28^{2} \approx 276 \mathrm{~m}^{-2}$, i.e. 276 drops per square meter) and (2) the expected portion of the surface area of the circle, with a center uniformly (randomly) distributed over the blotting paper, falling inside the boundaries of that blotting paper. The latter can be calculated analytically in a straightforward manner for circles with radii up to half the length of a side of the blotting paper, using the subdivision in regions $\mathrm{A}, \mathrm{B}$, and $\mathrm{C}$ as shown in Fig. 1. In Appendix A we show that the expected fraction of a square blotting paper with sides $L$ covered by a randomly located circle with radius $R$ equals

$$
\left\langle A_{0}\right\rangle=\pi r^{2}-\frac{8}{3} r^{3}+\frac{1}{2} r^{4},
$$

where $r=R / L$ is the normalized circle radius. Hence, $\left\langle A_{0}\right\rangle \times L^{2}$ is the expected overlap area between a circle with
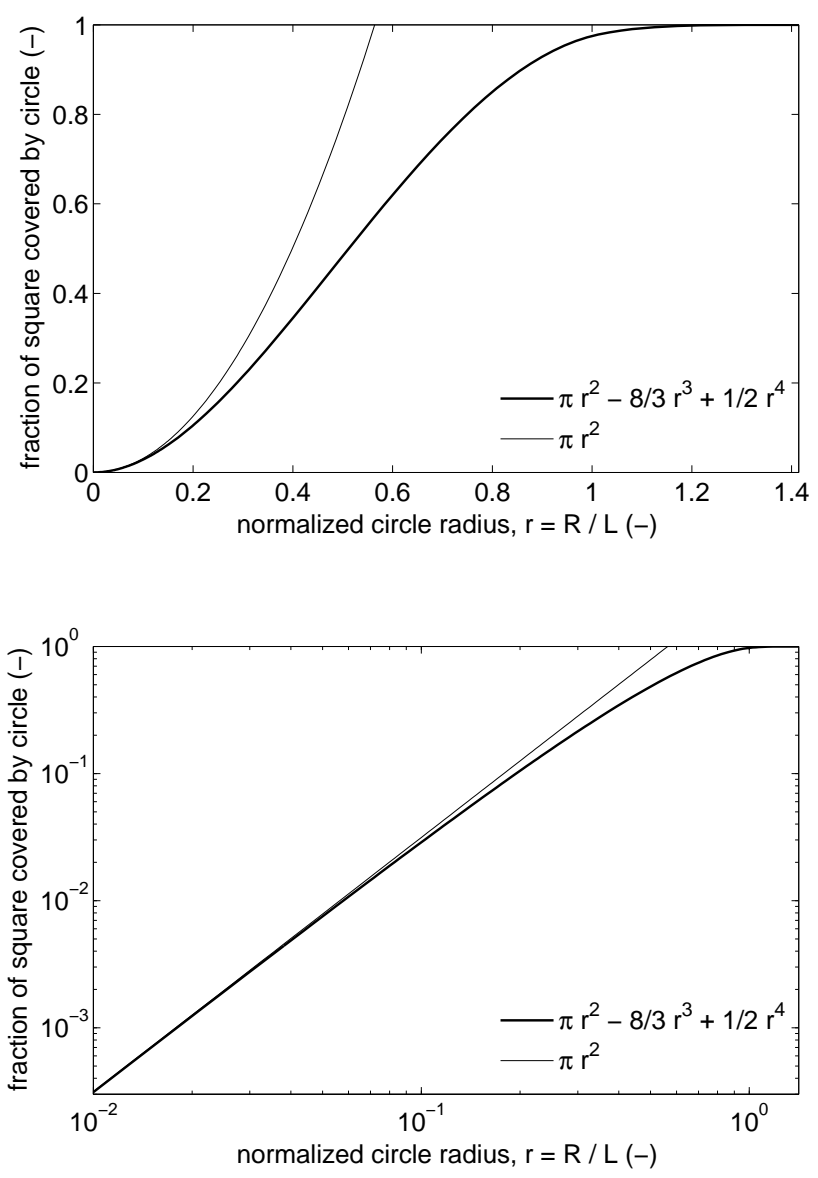

Fig. 2. Expected fraction of a square with sides $L$ covered by a circle with radius $R$, of which the center is uniformly distributed inside the square, as a function of the normalized circle radius $r=R / L$, with (bold line) and without (thin line) taking the edge effect into consideration. Top panel: linear axes; bottom panel: logarithmic axes. Note that the validity of our analytical solution (Eq. 1) is limited to the interval $0 \leq r \leq 1$ (the solution for the remaining interval $1 \leq r \leq \sqrt{2}$ is presented elsewhere).

radius $R$ and a square with sides $L$, when the circle center is uniformly (randomly) distributed inside the square.

Figure 2 shows, using linear as well as logarithmic axes, Eq. (1) for the interval $0 \leq r \leq \sqrt{2}$ (bold line), compared to the relation without correcting for the edge effect (thin line), i.e. just the first term on the right-hand side of Eq. (1). This first term simply represents the area of a circle with radius $r$, whereas the second and third terms on the right-hand side of Eq. (1) account for the edge effect. Although Eq. (1) has been derived in Appendix A assuming that the normalized circle radius belongs to the interval $0 \leq r \leq 1 / 2$, it can be demonstrated that its validity extends to $r \leq 1$.

Clearly, the expected surface area of the circle inside the blotting paper edges will eventually become equal to the area of the blotting paper itself, when the circle covers the paper entirely no matter where its center is located. This will 


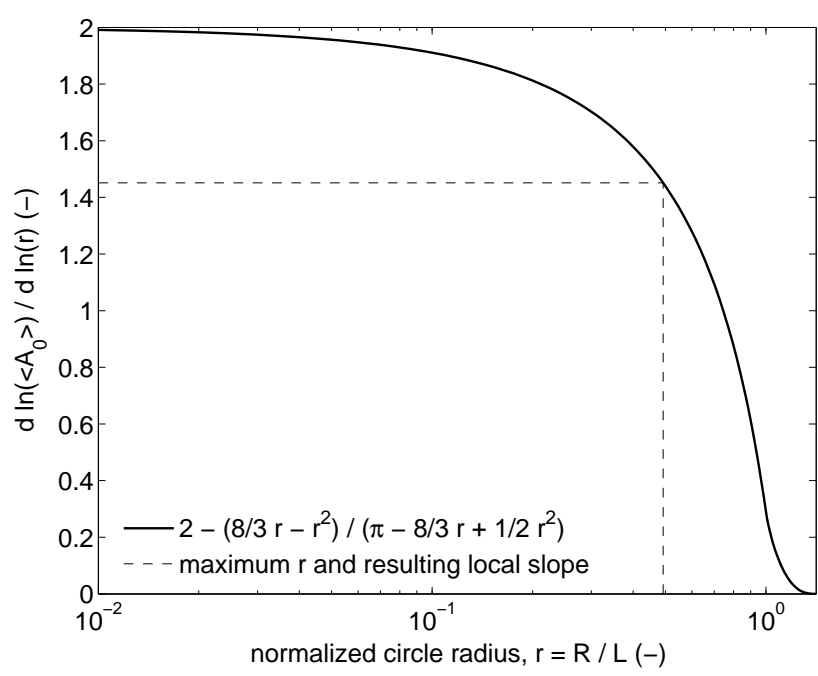

Fig. 3. The local slope of the bold line in the bottom panel of Fig. 2 as a function of the normalized circle radius $r=R / L$ (bold line). The dashed line indicates the maximum normalized circle radius considered by LS and the corresponding local slope. Note that the validity of our analytical solution (Eq. 2) is limited to the interval $0 \leq r \leq 1$ (the solution for the remaining interval $1 \leq r \leq \sqrt{2}$ is presented elsewhere).

happen if the circle radius exceeds $\sqrt{2}$ times the length of a side of the blotting paper (i.e. $r \geq \sqrt{2}$ ). As noted in Appendix $A$, for the interval $1 \leq r \leq \sqrt{2}$ we have been able to solve $\left\langle A_{0}\right\rangle$ analytically as well. However, the derivation of this expression is beyond the scope of this paper. The result is more complicated than Eq. (1) and will be presented elsewhere. Moreover, for the problem at hand, Eq. (1) covers all relevant values of $r$. Since for a Poisson process the expected number of raindrop stains in a circle with a given radius is proportional to the expected surface area of that circle falling inside the blotting paper boundary, multiplication of the ordinate of Fig. 2 with the (expected) total number of raindrop stains on the blotting paper yields the expected number of drop stains in the circle as a function of $r$.

The difference between the thin line and the bold line in Fig. 2, i.e. the expected portion of a circle with a given radius falling outside the blotting paper, provides a measure for the underestimation of the number of raindrop stains caused by the edge effect. For the largest radius considered by LS to fit a straight line to their Fig. 2 (corresponding to a normalized circle radius of $r=R / L=631 / 1280 \approx 0.5$ ), almost $40 \%$ of the circle area falls outside the blotting paper boundaries on average, indicating that the number of raindrop stains will be underestimated by about $40 \%$ for circles of this size (if the stains are uniformly distributed). It should be emphasized that this underestimation can be entirely explained as an edge effect in an otherwise homogeneous rainfall sample and does not require invoking any scaling hypothesis.
A direct appreciation of the edge effect on the value of the correlation dimension for homogeneously distributed raindrops may be obtained by taking the derivative of $\left\langle A_{0}\right\rangle$ with respect to the normalized circle radius $r=R / L$ on a log-log plot (i.e. the local slope of the bold line in the bottom panel of Fig. 2):

$\frac{\mathrm{d} \ln \left\langle A_{0}\right\rangle}{\mathrm{d} \ln r}=\frac{r}{\left\langle A_{0}\right\rangle} \frac{\mathrm{d}\left\langle A_{0}\right\rangle}{\mathrm{d} r}=2-\frac{8 r / 3-r^{2}}{\pi-8 r / 3+r^{2} / 2}$

$(0 \leq r \leq 1)$. This function is plotted in Fig. 3, which shows how the local slope of the bold line in the bottom panel of Fig. 2 decreases from 2 to 0 as a function of the normalized radius of the circle considered. The value of 2 corresponds to the "correlation dimension" expected for a homogeneous Poisson process in the absence of an edge effect. For the maximum circle radius considered by LS to determine the correlation dimension (corresponding to $r=R / L=631 / 1280 \approx 0.5)$, the local slope would be about 1.45 (dashed line in Fig. 3). The actual effect of the edge of the blotting paper will be less pronounced because the correlation dimension is not determined as the local slope but as the average slope over a certain range of circle radii, as will be shown in the next section.

\section{Blotting paper experiment revisited}

Ideally, we would have preferred to take the original data of the blotting paper experiment reported by LS, repeat their fractal analysis, and confront the outcome with the analytical solution to the edge effect presented in Sect. 3. However, unfortunately the original data (i.e. the raindrop stain coordinates) are no longer available (Gabella et al., 2001). In order to be able to obtain a quantitative appreciation of the statistical significance of the fractal correlation dimensions reported by LS, notwithstanding the absence of the original data, we have therefore proceeded as follows. We have digitized the individual raindrop stains indicated as small squares in Fig. 1a of Schertzer and Lovejoy (1989), which is an enlarged version of Fig. 1 of LS. In this manner, we have been able to recover 438 of the 452 drop stains that were supposedly present on the original blotting paper. The 438 individual raindrop coordinates (in $\mathrm{mm}$, given the $1280 \times 1280 \mathrm{~mm}^{2}$ size of the blotting paper) are shown in Fig. 4. The resolution with which the individual points have been digitized is approximately $2.15 \mathrm{~mm}$ in the $x$-direction and $2.35 \mathrm{~mm}$ in the $y$-direction.

Figure 5 shows how the fractal correlation dimension of the spatial point pattern of Fig. 4 is determined. The employed procedure is the same as that described by LS (see also Sect. 2): the correlation dimension is estimated as the slope of a straight line determined using linear regression on the logarithmic values, uniformly distributed over the radius interval in logarithmic space (10 values per decade, with a 


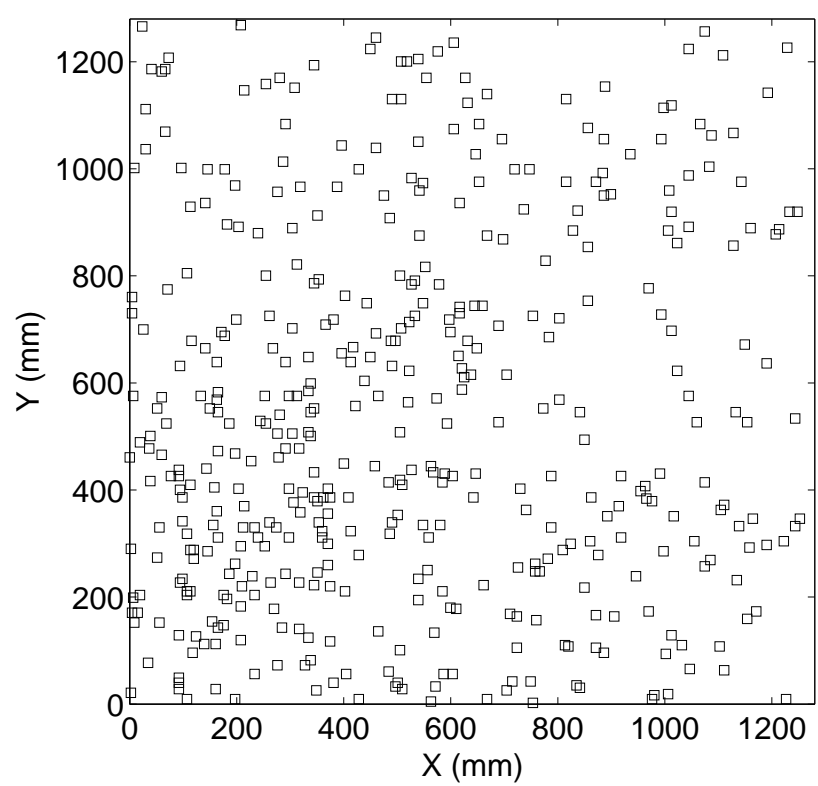

Fig. 4. Digitized version of Fig. 1a of Schertzer and Lovejoy (1989) (equivalent to Fig. 1 of LS) with the approximate locations of 438 of the original 452 raindrop stain centers collected on a $128 \times 128 \mathrm{~cm}^{2}$ chemically treated blotting paper during an exposure time of approximately $1 \mathrm{~s}$.

minimum circle radius of $10^{1.0}=10 \mathrm{~mm}$ and a maximum radius of $10^{2.8} \approx 631 \mathrm{~mm}$ ). In accordance with the analysis of LS (their Fig. 2), we have also included a comparison of our analytical solution with the average number of drops in equally logarithmically spaced annuli.

Our Fig. 5 closely corresponds to Fig. 2 of LS, the main difference being that LS obtain a fractal correlation dimension of 1.83 , whereas we find the slightly larger value of 1.92. This difference can partly be attributed to the 14 missing raindrop stains in our Fig. 4 as compared to their Fig. 1. Figure 5 also exhibits a lack of small distances as compared to Fig. 2 of LS, which may be partly due to the limited resolution with which we digitized their figure. This explains why we were forced to employ a minimum circle radius of $10 \mathrm{~mm}$, as opposed to the $3 \mathrm{~mm}$ used by LS, which may be another reason why our estimated correlation dimension is larger than that of LS. Nevertheless, our value is still smaller than 2, potentially indicating a fractal clustering behavior of the spatial distribution of raindrops.

To test the statistical significance of the obtained value given the finite resolution with which the individual raindrop stains have been digitized, we have generated 1000 replicates of Fig. 4, assuming the $x$ - and $y$-coordinates of the stain centers to be independent uniformly distributed random variables with means corresponding to the digitized values and ranges corresponding to the resolutions indicated above ( $2.15 \mathrm{~mm}$ in the $x$-direction and $2.35 \mathrm{~mm}$ in the $y$-direction).

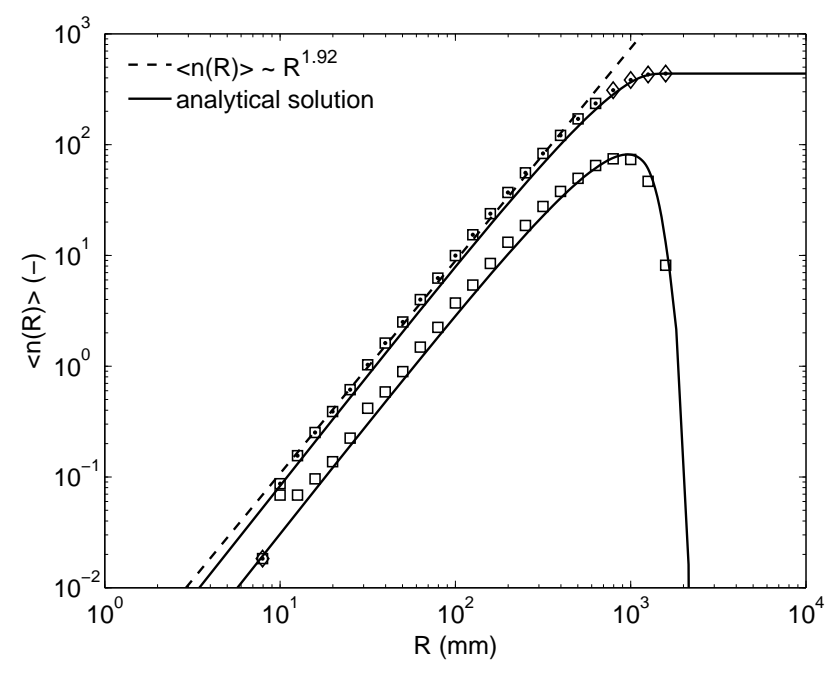

Fig. 5. Log-log plot of the average number of other drops $\langle n(R)\rangle$ in a radius $R$ around each drop for the spatial point pattern of Fig. 4 (filled squares and diamonds). The resulting fractal correlation dimension of 1.92 has been determined using linear regression on the logarithmic values for circle radii between $10 \mathrm{~mm}$ and $63 \mathrm{~cm}$ (indicated by the dashed line passing through the filled squares). The open squares indicate the average number of drops in equally logarithmically spaced annuli (corresponding to the differences between subsequent filled squares or diamonds). The bold lines indicate our analytical solution to $\langle n(R)\rangle$ for homogeneously distributed raindrops, including the edge effect (Eq. 1, Fig. 2).

Some statistics of the 1000 exponents determined in this manner are given in Table 1 (2nd column, "LS (1/1)"). The uncertainty due to the finite resolution associated with the digitization process is seen to be small.

However, the most striking result of Fig. 5 is that our analytical solution to the edge effect in homogeneous Poissonian rain (Eq. 1) closely follows the experimentally determined average raindrop stain counts as a function of increasing search radius, both for the circles (the upper bold line) and for the annuli (the lower bold line). This suggests that the edge effect identified above could be the main reason for the observed correlation dimension being smaller than 2. This is consistent with earlier observations by Gabella et al. (2001), who concluded on the basis of a limited Monte Carlo simulation study that "the correlation dimension estimated from the raindrop distribution observed by Lovejoy and Schertzer could be compatible with a uniform random spatial distribution".

\section{Monte Carlo experiment}

To investigate the statistical significance of the estimated correlation dimension in a more rigorous manner, we have generated 1000 realizations of a homogeneous spatial Poisson point process, each mimicking the original experimental 
Table 1. Statistics (means, standard deviations, and the 5th, 25th, 50th, 75th and 95th percentiles) of the probability distribution of the fractal correlation dimension determined for 1000 independent randomly generated replicates. The replicates of the supposedly clustered spatial point pattern of LS (Fig. 4) represent the uncertainty associated with the coordinates of the raindrop stains as a result of the digitization process. Those of the uniform spatial point pattern represent the uncertainty associated with the coordinates of simulated raindrop stains obeying a homogeneous Poisson point process in space with the same number density as Fig. 4. Columns 2 and 3 (indicated as " $(1 / 1)$ ") correspond to the entire $1.28 \times 1.28 \mathrm{~m}^{2}$ square, whereas columns 4 and 5 (indicated as "(1/9)") correspond to the central square, after the entire square has been subdivided into nine equal squares.

\begin{tabular}{ccccc}
\hline Statistic & LS (1/1) & Poisson (1/1) & LS (1/9) & Poisson (1/9) \\
\hline mean & 1.9226 & 1.9155 & 2.0287 & 2.0204 \\
s.d. & 0.0077 & 0.0428 & 0.0202 & 0.1002 \\
$5 \%$ & 1.9110 & 1.8495 & 2.0019 & 1.8643 \\
$25 \%$ & 1.9174 & 1.8859 & 2.0129 & 1.9532 \\
$50 \%$ & 1.9219 & 1.9131 & 2.0263 & 2.0120 \\
$75 \%$ & 1.9274 & 1.9447 & 2.0407 & 2.0791 \\
$95 \%$ & 1.9362 & 1.9884 & 2.0671 & 2.1873 \\
\hline
\end{tabular}

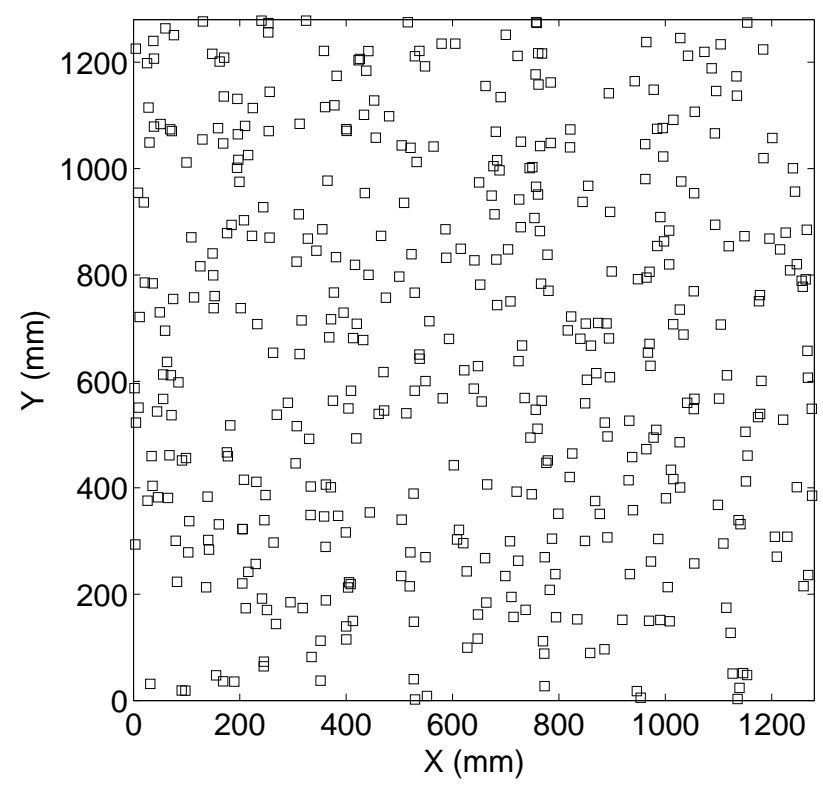

Fig. 6. One realization of 438 uniformly distributed raindrop stain centers on a $128 \times 128 \mathrm{~cm}^{2}$ square, the homogeneous (Poissonian) equivalent of Fig. 4.

setup of LS. Given the fixed number of 438 raindrop stains on the blotting paper, the Poisson homogeneity hypothesis dictates that the coordinates of the 438 stain centers are independent identically distributed random variables following a

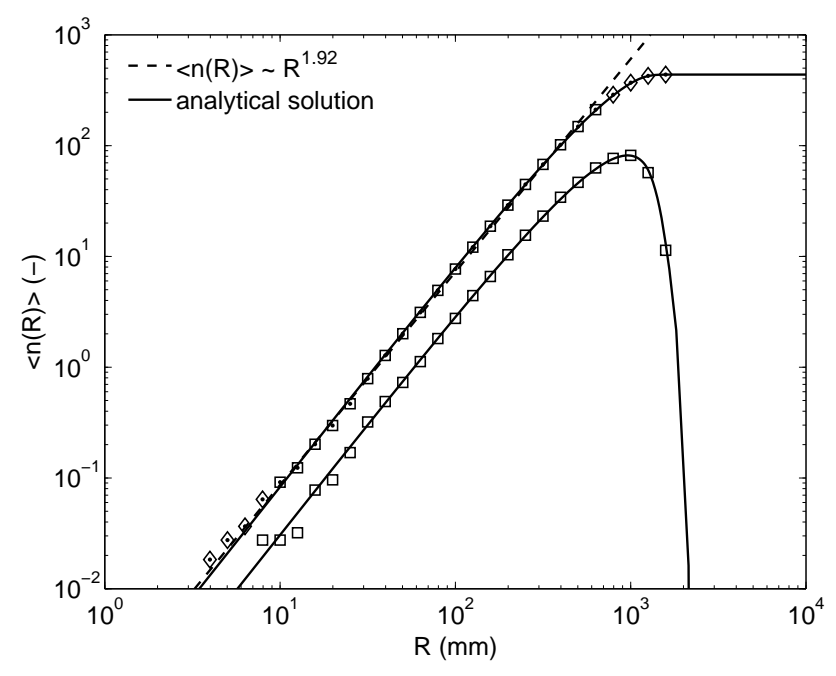

Fig. 7. The same analysis as Fig. 5, but applied to the spatial point pattern of Fig. 6. Note that the fractal correlation dimension determined for this homogeneous realization (1.92) is the same as that for the supposedly fractal point pattern of Fig. 4 .

uniform probability distribution (Cox and Isham, 1980). Figure 6 shows one of the generated realizations and Fig. 7 the corresponding fractal analysis. These figures are the homogeneous equivalents of Figs. 4 and 5, respectively. Note that the correspondence between theory and (numerical) experiment is excellent (Fig. 7), demonstrating the usefulness of our analytical solution. Also note that the fractal correlation dimension determined for the homogeneous realization of Fig. 6 (1.92) is the same as that determined previously for the supposedly fractal point pattern of Fig. 4. This is a coincidence, however, as the correlation dimension will vary from one realization to the next.

Exactly how variable the determined correlation dimensions are, is shown in Table 1 (3rd column, "Poisson (1/1)"), which provides some summary statistics of the probability distribution of the estimated correlation dimension. It can be seen that both the mean and the median of the correlation dimension are actually very close to the previously mentioned value of 1.92 . The distribution is seen to be nearly symmetrical, with $90 \%$ of the probability mass concentrated in the interval between 1.85 and 1.99. This shows that a value of 1.92 for the correlation dimension cannot be considered as an unequivocal proof for the fractal nature of the microstructure of rain. Such a value is not significantly different (in a statistical sense) from values that would be expected for a homogeneous spatial raindrop distribution over a square of finite size. Edge effects alone are sufficient to explain such "fractal" correlation dimensions.

Figure 8, finally, shows the influence of the maximum circle radius considered on the estimation of the correlation dimension. In accordance with LS, slopes have been obtained using linear regression on the logarithmic values, 
uniformly distributed over the interval in logarithmic space (10 values per decade, with a minimum circle radius of $10 \mathrm{~mm}$ and a maximum radius of $63 \mathrm{~cm}$ ). Figure 8 shows that, apparently, the influence of the blotting paper's finite size remains appreciable even for circles which are an order of magnitude smaller than the paper itself: the mean correlation dimensions in Fig. 8 (indicated by the circles) remain smaller than the limiting value of 2 , even for the smallest maximum radii considered.

The indicated slopes represent "apparent" correlation dimensions, as their fractal values are entirely the result of a sampling artifact (edge effect). Interestingly, the correlation dimensions determined on the basis of Fig. 4 (i.e. the crosses in Fig. 8) become larger than the Poissonian value of 2 for maximum circle radii smaller than about $20 \mathrm{~cm}$. The average correlation dimensions for homogeneously distributed raindrops (indicated by the circles) never exceed the limiting value of 2, although values in excess of 2 may be reached for individual realizations. Nevertheless, the crosses always fall within the 50\% confidence limits of the homogeneous correlation dimensions, confirming our previous observation that the spatial point pattern of Fig. 4 does not exhibit a statistically significant departure from Poissonian homogeneity (in any case, not as far as its correlation dimension is considered). Alternative ways to test for (the absence of) complete spatial randomness in two-dimensional point patterns (e.g., Cressie, 1991) are beyond the scope of this paper.

\section{Discussion and conclusions}

In conclusion, the claim by LS that their blotting paper experiment provides empirical evidence for the fractal hypothesis that "drops are (hierarchically) clustered" and that "inhomogeneity in rain is likely to extend down to millimeter scales" needs to be reconsidered. Our analytical results confirm previously reported Monte Carlo simulations (Jameson and Kostinski, 1998; Gabella et al., 2001) showing that as a result of instrumental artifacts (edge effects) the empirical results presented by LS are not statistically significant enough to reject the Poisson homogeneity hypothesis in favor of a fractal description of the discrete nature of rainfall. As such our analytical solution (Eq. (1)) provides an explanation for what Gabella et al. (2001) and Gabella and Perona (2001) call the "systematic error" in the statistical analysis of the blotting paper experiment, i.e. the negative bias in the estimated correlation dimension resulting from edge effects. Through Monte Carlo simulations we have also considered the "random error" resulting from the finite sample size, i.e. uncertainties in the estimated correlation dimension due to random fluctuations in the total number of raindrop stains on the blotting paper from one realization to the next. In more general terms, it seems that the derived expression (Eq. (1)) can be used as a reference curve against which to

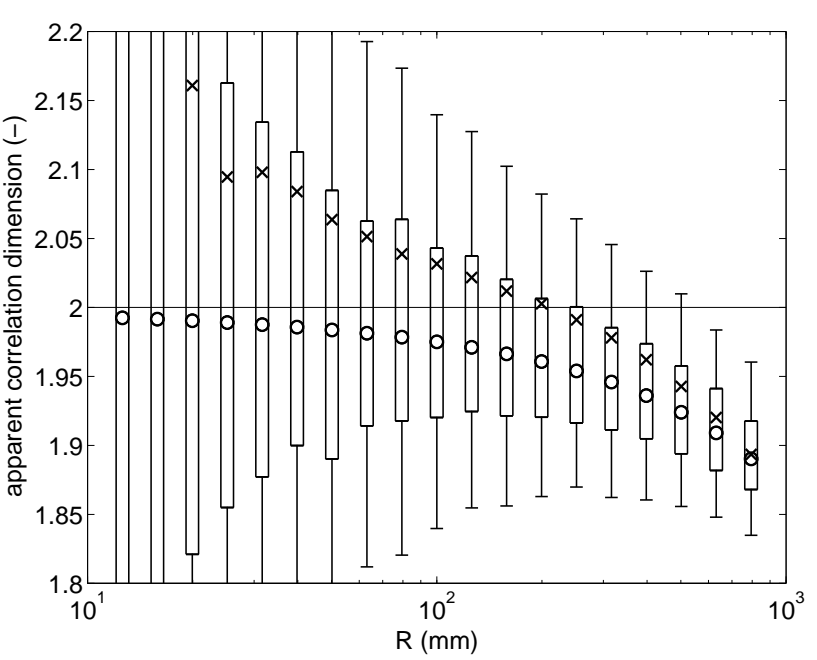

Fig. 8. Box and whiskers plot of the apparent fractal correlation dimension as a function of the maximum circle radii used to perform the regression analyses. The circles correspond to the mean correlation dimensions for 1000 homogeneous realizations such as the one shown in Fig. 6. The boxes show the corresponding 25th and 75 th percentiles, whereas the whiskers show the 5th and 95th percentiles. The crosses correspond to the correlation dimensions determined on the basis of Fig. 4. The second to last cross and circle correspond to the maximum circle radius employed in Figs. 5 and 7 , respectively.

test the statistical significance of fractal correlation dimensions determined from spatial point patterns.

In future experiments, the negative bias in the estimated correlation dimension due to systematic edge effects may be reduced by increasing the area of the blotting paper while keeping the maximum circle radius fixed (thereby reducing the ratio $R / L$ ) and/or by keeping a guard area inside the perimeter of the blotting paper, as suggested by Gabella et al. (2001) ${ }^{1}$. If we subdivide the entire blotting paper into nine equal squares and only consider the central square in the calculation of the correlation dimension (i.e. keeping a guard area of $1280 / 3 \approx 427 \mathrm{~mm}$ and limiting the maximum circle radius to $10^{2.6} \approx 398 \mathrm{~mm}$ ) the edge effect disappears, apart from some remaining sampling variability (see Table 1, 4th and 5th columns, "LS (1/9)" and "Poisson (1/9)", respectively). The resulting average correlation dimensions for the supposedly fractal spatial point pattern of Fig. 4 and for the

\footnotetext{
${ }^{1}$ In a study concerned with the statistical analysis of spatial patterns of rainfall cells Bacchi et al. (1996) employed a similar guard area. They also consider alternative measures to test for complete spatial randomness of point patterns. This shows that the statistical results presented in this paper are not restricted to the discrete microstructure of rainfall, but may also be relevant at much larger (meso- $\beta$ or $-\gamma$ ) scales. Leonard et al. (2006) presented an analysis of edge effects in simulated spatial point patterns of rain cells at such scales.
} 
corresponding homogeneous (uniform) Poisson case are very close to 2 , the value expected for raindrop stains produced by a homogeneous Poisson process unaffected by edge effects.

The most appropriate manner to describe the discrete nature of rainfall is still the topic of ongoing debates (e.g., Kostinski et al., 2006; Lilley et al., 2006; Jameson, 2007). However, that the seemingly conflicting approaches of generalized Poisson process models versus multi-fractal models do not necessarily have to exclude each other has been argued by Fabry (1996) and more recently theoretically investigated by Lovejoy and Schertzer (2006, 2008). The hypothesis supporting this reconciliation is that gravitational mixing (associated with the differential fall speeds of the raindrops) tends to lead to homogeneous rainfall at the smallest spatial scales, whereas (fractal) scaling regimes exist at larger scales. Indeed, spatial distributions of raindrops measured using stereo-photography indicate that the multi-fractal character of rain would be more clearly detectable in the spatial distribution of the liquid (rain)water content than in the spatial distribution of the drops per se - which has been found to be relatively homogeneous at small scales (DesaulniersSoucy et al., 2001; Lovejoy et al., 2003; Lilley et al., 2006).

\section{Appendix A}

\section{Expected overlap between a circle and a square, when the circle center is randomly distributed inside the square}

In this appendix, we derive an analytical expression for the expected overlap between a circle and a square, when the circle center is uniformly distributed inside the square. This is a problem in geometrical probability (e.g., Kendall and Moran, 1963; Santaló, 1979) and stochastic geometry (e.g., Stoyan et al., 1987). Without loss of generality, we assume the square to have unit area. We limit ourselves to the case where the circle radius $r$ is smaller than $1 / 2$, which guarantees that the disk defined by the circle will cross at most two sides of the unit square ${ }^{2}$.

Consider the subdivision in regions $\mathrm{A}, \mathrm{B}$, and $\mathrm{C}$ as shown in Fig. 1. Regions B and $\mathrm{C}$ define a border of size $r$ inside the square. Therefore, as long as the circle center falls inside region A, the circle will fall entirely inside the square. The probability that a point uniformly distributed inside the unit square falls inside region $\mathrm{A}$ is equal to the area of $\mathrm{A}$, namely $(1-2 r)^{2}$. The overlap between the circle and the

\footnotetext{
${ }^{2} \mathrm{~A}$ related problem is that of the expected area of overlap between a circle of radius $r$ and a unit square, when the circle center is uniformly distributed in a region consisting of all points in the plane whose distance from the square is not greater than $r$. The area of this region is $1+4 r+\pi r^{2}$ and the area of the circle is $\pi r^{2}$. The resulting expected area of overlap equals the probability of any point in the unit square being covered by the circle, i.e. $\pi r^{2} /\left(1+4 r+\pi r^{2}\right)$ (Garwood, 1947; Kendall and Moran, 1963; Santaló, 1979).
}

square, given that the circle center is located inside region $\mathrm{A}$, is then $\pi r^{2}$.

If the circle center falls in region $\mathrm{B}$, the circle will fall partly outside the square, but on one side only. The probability that a point uniformly distributed inside the unit square will fall inside region $\mathrm{B}$ is equal to the area of $\mathrm{B}$, i.e. $4 r(1-2 r)$. Suppose the center of a circle falls inside region $\mathrm{B}$ and the (horizontal or vertical) distance from the circle center to the boundary of the square is $y$. Then the area of the circle falling outside the square as a function of $y$ $(0 \leq y \leq r)$ is

$A_{1}(y)=2 \int_{0}^{\sqrt{r^{2}-y^{2}}}\left(\sqrt{r^{2}-x^{2}}-y\right) \mathrm{d} x$.

If the circle center is uniformly distributed in region $\mathrm{B}$ then the distance $y$ from the circle center to the boundary of the square is a random variable uniformly distributed over the interval $[0, r]$. The expected circle area falling outside the square, given that the circle center is located inside region B, is then

$$
\begin{aligned}
\left\langle A_{1}\right\rangle= & \frac{1}{r} \int_{0}^{r} A_{1}(y) \mathrm{d} y \\
= & \frac{2}{r} \int_{0}^{r} \int_{0}^{\sqrt{r^{2}-y^{2}}}\left(\sqrt{r^{2}-x^{2}}-y\right) \mathrm{d} x \mathrm{~d} y \\
= & \frac{2}{r} \int_{0}^{r} \int_{0}^{\sqrt{r^{2}-y^{2}}} \sqrt{r^{2}-x^{2}} \mathrm{~d} x \mathrm{~d} y- \\
& \frac{2}{r} \int_{0}^{r} \int_{0}^{\sqrt{r^{2}-y^{2}}} y \mathrm{~d} x \mathrm{~d} y,
\end{aligned}
$$

where $\langle\cdot\rangle$ is the expectation operator. Changing the integration order yields

$$
\begin{aligned}
\left\langle A_{1}\right\rangle= & \frac{2}{r} \int_{0}^{r} \int_{0}^{\sqrt{r^{2}-x^{2}}} \sqrt{r^{2}-x^{2}} \mathrm{~d} y \mathrm{~d} x- \\
& \frac{2}{r} \int_{0}^{r} \int_{0}^{\sqrt{r^{2}-x^{2}}} y \mathrm{~d} y \mathrm{~d} x \\
= & \frac{2}{r} \int_{0}^{r}\left(r^{2}-x^{2}\right) \mathrm{d} x-\frac{1}{r} \int_{0}^{r}\left(r^{2}-x^{2}\right) \mathrm{d} x \\
= & \frac{1}{r} \int_{0}^{r}\left(r^{2}-x^{2}\right) \mathrm{d} x=\frac{2}{3} r^{2} .
\end{aligned}
$$

Hence, the expected overlap between the circle and the square, given that the circle center is located inside region B, is $(\pi-2 / 3) r^{2}$.

If the circle center falls in region $\mathrm{C}$, the circle will again fall partly outside the square, but now on two sides (see Fig. 1). The probability that a point uniformly distributed inside the unit square will fall inside region $\mathrm{C}$ is equal to the area of C, i.e. $4 r^{2}$.

Because the circle center is assumed to be uniformly distributed inside the square, its horizontal and vertical positions 
inside the square (and consequently in each of the three regions identified in Fig. 1) are independent. Therefore, the areas of the two fractions of the circle falling outside the square, if the circle center is located in region $\mathrm{C}$, are independent. Hence, the expected circle area falling outside the square, given that the circle center is located inside region $\mathrm{C}$, is twice that given by Eq. (A3) minus the expected area of the overlap between the two fractions of the circle falling outside the square. These fractions will only overlap if the circle center is located at a distance less than $r$ from the corners of the square, i.e. in that part of region $\mathrm{C}$ between the dashed circle segments in Fig. 1 and each of the corners of the square. If the circle center is uniformly distributed inside the unit square, the probability of this event is $\pi r^{2}$. Hence, given that the circle center is located in region $\mathrm{C}$, this (conditional) probability is $\pi / 4$.

Suppose the center of a circle falls inside region $\mathrm{C}$ and the horizontal and vertical distances from the circle center to the boundary of the square are $x$ and $y$, respectively. Then the area of the overlapping fractions of the circle falling outside the square as a function of $x$ and $y\left(\sqrt{x^{2}+y^{2}} \leq r\right)$ is

$A_{2}(x, y)=\int_{x}^{\sqrt{r^{2}-y^{2}}}\left(\sqrt{r^{2}-s^{2}}-y\right) \mathrm{d} s$.

If the circle center is uniformly distributed in the part of region $\mathrm{C}$ between the dashed circle segments and the corners of the square (see Fig. 1), then the horizontal distance $x$ and the vertical distance $y$ from the circle center to the boundary of the square are independent uniformly distributed random variables such that $\sqrt{x^{2}+y^{2}} \leq r$. The expected area of the overlapping fractions of the circle falling outside the square, given that the circle center is located in the part of region $\mathrm{C}$ between the dashed circle segments and the corners of the square, is then

$$
\begin{aligned}
\left\langle A_{2}\right\rangle= & \frac{4}{\pi r^{2}} \int_{0}^{r} \int_{0}^{\sqrt{r^{2}-y^{2}}} A_{2}(x, y) \mathrm{d} x \mathrm{~d} y \\
= & \frac{4}{\pi r^{2}} \int_{0}^{r} \int_{0}^{\sqrt{r^{2}-y^{2}}} \int_{x}^{\sqrt{r^{2}-y^{2}}} \sqrt{r^{2}-s^{2}} \mathrm{~d} s \mathrm{~d} x \mathrm{~d} y \\
& -\frac{4}{\pi r^{2}} \int_{0}^{r} \int_{0}^{\sqrt{r^{2}-y^{2}}} \int_{x}^{\sqrt{r^{2}-y^{2}}} y \mathrm{~d} s \mathrm{~d} x \mathrm{~d} y .
\end{aligned}
$$

Changing the integration order yields

$$
\begin{aligned}
\left\langle A_{2}\right\rangle= & \frac{4}{\pi r^{2}} \int_{0}^{r} \int_{0}^{\sqrt{r^{2}-y^{2}}} \int_{0}^{s} \sqrt{r^{2}-s^{2}} \mathrm{~d} x \mathrm{~d} s \mathrm{~d} y- \\
& \frac{4}{\pi r^{2}} \int_{0}^{r} \int_{0}^{\sqrt{r^{2}-y^{2}}} \int_{0}^{s} y \mathrm{~d} x \mathrm{~d} s \mathrm{~d} y \\
= & \frac{4}{\pi r^{2}} \int_{0}^{r} \int_{0}^{\sqrt{r^{2}-y^{2}}} s \sqrt{r^{2}-s^{2}} \mathrm{~d} s \mathrm{~d} y- \\
& \frac{4}{\pi r^{2}} \int_{0}^{r} \int_{0}^{\sqrt{r^{2}-y^{2}}} s y \mathrm{~d} s \mathrm{~d} y .
\end{aligned}
$$

Changing the integration order once more yields

$$
\begin{aligned}
\left\langle A_{2}\right\rangle= & \frac{4}{\pi r^{2}} \int_{0}^{r} \int_{0}^{\sqrt{r^{2}-s^{2}}} s \sqrt{r^{2}-s^{2}} \mathrm{~d} y \mathrm{~d} s- \\
& \frac{2}{\pi r^{2}} \int_{0}^{r} s\left(r^{2}-s^{2}\right) \mathrm{d} s \\
= & \frac{2}{\pi r^{2}} \int_{0}^{r} s\left(r^{2}-s^{2}\right) \mathrm{d} s=\frac{r^{2}}{2 \pi} .
\end{aligned}
$$

If the circle center is located in region $\mathrm{C}$, but at a distance larger than $r$ from the nearest corner of the square, then the two parts of the circle falling outside the square do not overlap. Hence, to obtain the expected area of the overlapping fractions of the circle falling outside the square, given that the circle center is located in region C, Eq. (A7) needs to be multiplied by the probability that the distance from the circle center to the nearest corner is less than $r$, given that the circle center is located in region C, i.e. $\pi / 4$. The resulting area is $r^{2} / 8$. As a consequence, the expected overlap between the circle and the square, given that the circle center is located inside region $C$, is $(\pi-4 / 3+1 / 8) r^{2}$ or $(\pi-29 / 24) r^{2}$.

Finally, the expected overlap between a circle and a square, when the circle center is randomly distributed inside the square is (for $0 \leq r \leq 1 / 2$ )

$$
\begin{aligned}
\left\langle A_{0}\right\rangle= & (1-2 r)^{2} \pi r^{2}+4 r(1-2 r)\left(\pi-\frac{2}{3}\right) r^{2}+ \\
& 4 r^{2}\left(\pi-\frac{4}{3}+\frac{1}{8}\right) r^{2} \\
= & \pi r^{2}-\frac{8}{3} r^{3}+\frac{1}{2} r^{4} .
\end{aligned}
$$

It can be demonstrated that the validity of Eq. (A8) extends to $r \leq 1$. Moreover, we have been able to obtain an analytical solution for the interval $1 \leq r \leq \sqrt{2}$ as well. However, these derivations are beyond the scope of this paper and will be presented elsewhere. Note that Eq. (A8) can also be obtained from the probability density function of the distance between random point pairs inside a square (Garwood, 1947; Kagan, 2007).

Acknowledgements. The work reported in this paper has been supported financially by EU projects VOLTAIRE (EVK2-2001-00273) and FLOODsite (GOCE-CT-2004-505420). R. U. acknowledges the EU for a Marie Curie Postdoctoral Fellowship (ENV4-CT965030), the Grup de Recerca Aplicada en Hidrometeorologia (GRAHI) of the Universitat Politècnica de Catalunya, Barcelona, Spain, for a visiting professorship in the framework of a Distinció de Recerca de la Generalitat de Catalunya, and the Netherlands Organization for Scientific Research (NWO) for a grant (016.021.003) in the framework of the Innovational Research Incentives Scheme (Vernieuwingsimpuls). In addition, R. U. thanks Paul Torfs for pointing out some references and Marie-Colette van Lieshout (CWI) for checking the derivation in the appendix.

Edited by: J. de Lima

Reviewed by: M. Larsen and S. Lovejoy 


\section{References}

Bacchi, B., Ranzi, R., and Borga, M.: Statistical characterization of spatial patterns of rainfall cells in extratropical cyclones, J. Geophys. Res., (D101), 26277-26286, 1996.

Cornford, S. G.: Sampling errors in measurements of raindrop and cloud droplet concentrations, Meteorol. Mag., 96, 271-282, 1967.

Cox, D. R. and Isham, V.: Point processes, Chapman \& Hall, London, 188 pp., 1980

Cressie, N. A. C.: Statistics for spatial data, John Wiley \& Sons, New York, 936 pp., 1991.

Desaulniers-Soucy, N., Lovejoy, S., and Schertzer, D.: The HYDROP experiment: An empirical method for the determination of the continuum limit in rain, Atmos. Res., 59-60, 163-197, 2001

Fabry, F.: On the determination of scale ranges for precipitation fields, J. Geophys. Res., 101(D8), 12819-12826, 1996.

Gabella, M. and Perona, G.: Comments on "Errors in the estimate of the fractal correlation dimension of raindrop spatial distribution" - Reply, J. Appl. Meteorol., 40, 2099-2099, 2001.

Gabella, M., Pavone, S., and Perona, G.: Errors in the estimate of the fractal correlation dimension of raindrop spatial distribution, J. Appl. Meteorol., 40, 664-668, 2001.

Garwood, F.: The variance of the overlap of geometrical figures with reference to a bombing problem, Biometrika, 34, 1-17, 1947.

Gertzman, H. R. and Atlas, D.: Sampling errors in the measurement of rain and hail parameters, J. Geophys. Res., 82, 4955-4966, 1977.

Grassberger, P. and Procaccia, I.: Characterization of strange attractors, Phys. Rev. Lett., 50, 346-349, 1983a.

Grassberger, P. and Procaccia, I.: Measuring the strangeness of strange attractors, Physica D, 9, 189-208, doi:10.1016/01672789(83)90 298-1, 1983b.

Jameson, A. R.: A new characterization of rain and clouds: Results from a statistical inversion of count data, J. Atmos. Sci., 64, 2012-2028, doi:10.1175/JAS3950.1, 2007.

Jameson, A. R. and Kostinski, A. B.: Fluctuation properties of precipitation. Part II: Reconsideration of the meaning and measurement of raindrop size distributions, J. Atmos. Sci., 55, 283-294, 1998.

Jameson, A. R. and Kostinski, A. B.: What is a raindrop size distribution?, B. Am. Meteorol. Soc., 82, 1169-1177, 2001 a.

Jameson, A. R. and Kostinski, A. B.: Comments on "Errors in the estimate of the fractal correlation dimension of raindrop spatial distribution", J. Appl. Meteorol., 40, 2098-2098, 2001b.

Joss, J. and Waldvogel, A.: Raindrop size distribution and sampling size errors, J. Atmos. Sci., 26, 566-569, 1969.

Kagan, Y. Y.: Earthquake spatial distribution: the correlation dimension, Geophys. J. Int., 168, 1175-1194, doi:10.1111/j.1365246X.2006.03 251.x, 2007.

Kendall, M. G. and Moran, P. A. P.: Geometrical probability, Charles Griffin, London, 102-118, 1963.

Kostinski, A. B. and Jameson, A. R.: Fluctuation properties of precipitation. Part I: On deviations of single-size drop counts from the Poisson distribution, J. Atmos. Sci., 54, 2174-2186, 1997.

Kostinski, A. B. and Shaw, R. A.: Fluctuations and luck in droplet growth by coalescence, B. Am. Meteorol. Soc., 86, 235-244, 2005.
Kostinski, A. B., Larsen, M. L., and Jameson, A. R.: The texture of rain: Exploring stochastic micro-structure at small scales, J. Hydrol., 328, 38-45, doi:10.1016/j.jhydrol.2005.11.035, 2006.

Larsen, M. L., Kostinski, A. B., and Tokay, A.: Observations and analysis of uncorrelated rain, J. Atmos. Sci., 62, 4071-4083, 2005.

Lavergnat, J. and Golé, P.: A stochastic raindrop time distribution model, J. Appl. Meteorol., 37, 805-818, 1998.

Leonard, M., Metcalfe, A. V., and Lambert, M. F.: Efficient simulation of a space-time Neyman-Scott rainfall model, Water Resour. Res., 42, W11503, doi:10.1029/2006WR004 986, 2006.

Lilley, M., Lovejoy, S., Desaulniers-Soucy, N., and Schertzer, D.: Multifractal large number of drops limit in rain, J. Hydrol., 328 , 20-37, doi:10.1016/j.jhydrol.2005.11.063, 2006.

Lovejoy, S. and Schertzer, D.: Fractals, raindrops and resolution dependence of rain measurements, J. Appl. Meteorol., 29, 1167 1170, 1990a.

Lovejoy, S. and Schertzer, D.: Multifractals, universality classes and satellite and radar measurements of cloud and rain fields, J. Geophys. Res., 95(D3), 2021-2034, 1990b.

Lovejoy, S. and Schertzer, D.: Stereophotography of rain drops and compound Poisson - cascade processes, in: Preprints of the 12th Conference on Cloud Physics, 4.4.1-14.4.19, American Meteorological Society, Boston, 2006.

Lovejoy, S. and Schertzer, D.: Turbulence, rain drops and the $l^{1 / 2}$ number density law, New J. Phys., 10, doi:10.1088/13672630/10/7/075017, 2008.

Lovejoy, S., Lilley, M., Desaulniers-Soucy, N., and Schertzer, D.: Large particle number limit in rain, Phys. Rev. E, 68, 025301-14, 2003.

Marshall, J. S. and Hitschfeld, W.: Interpretation of the fluctuating echo from randomly distributed scatterers. Part I, Can. J. Phys., 31, 962-994, 1953.

Marshall, J. S. and Palmer, W. M.: The distribution of raindrops with size, J. Meteorol., 5, 165-166, 1948.

Porrà, J. M., Sempere Torres, D., and Creutin, J.-D.: Modeling of drop size distribution and its applications to rainfall measurements from radar, in: Stochastic methods in hydrology: Rain, landforms and floods, edited by: Gupta, V. K., BarndorffNielsen, O. E., Perez-Abreu, V., and Waymire, E., 73-84, World Scientific, Singapore, 1998.

Pruppacher, H. R. and Klett, J. D.: Microphysics of clouds and precipitation, Reidel, Dordrecht, The Netherlands, 714 pp., 1978.

Rogers, R. R. and Yau, M. K.: A short course in cloud physics, Butterworth-Heinemann, Oxford, third edn., 290 pp., 1996.

Santaló, L. A.: Integral geometry and geometric probability, Addison-Wesley, Reading, 2nd edn., 404 pp., 1979.

Sasyo, Y.: On the probabilistic analysis of precipitation particles, in: Proceedings of the International Conference on Cloud Physics, 254-259, International Association of Meteorological and Atmospheric Physics, 1965.

Schertzer, D. and Lovejoy, S.: Generalized scale invariance and multiplicative processes in the atmosphere, Pure Appl. Geophys., 130, 57-81, 1989.

Smith, J. A.: Marked point process models of raindrop-size distributions, J. Appl. Meteorol., 32, 284-296, 1993.

Smith, P. L., Liu, Z., and Joss, J.: A study of sampling-variability effects in raindrop size observations, J. Appl. Meteorol., 32, 1259$1269,1993$. 
Stoyan, D., Kendall, W. S., and Mecke, J.: Stochastic geometry and its applications, Akademie-Verlag, Berlin, 122-131, 1987.

Theiler, J.: Efficient algorithm for estimating the correlation dimension from a set of discrete points, Phys. Rev. A, 36, 4456-4462, 1987.

Uijlenhoet, R. and Sempere Torres, D.: Measurement and parameterization of rainfall microstructure, J. Hydrol., 328, 1-7, doi:10.1016/j.jhydrol.2005.11.038, 2006.

Uijlenhoet, R., Stricker, J. N. M., Torfs, P. J. J. F., and Creutin, J.D.: Towards a stochastic model of rainfall for radar hydrology: Testing the Poisson homogeneity hypothesis, Phys. Chem. Earth (B), 24, 747-755, 1999.
Uijlenhoet, R., Porrà, J. M., Sempere Torres, D., and Creutin, J.-D.: Analytical solutions to sampling effects in drop size distribution measurements during stationary rainfall: Estimation of bulk rainfall variables, J. Hydrol., 328, 65-82, doi:10.1016/j.jhydrol.2005.11.043, 2006.

Wallace, P. R.: Interpretation of the fluctuating echo from randomly distributed scatterers. Part II, Can. J. Phys., 31, 995-1009, 1953.

Zawadzki, I.: Is rain fractal?, in: New Uncertainty Concepts in Hydrology and Water Resources, edited by: Kundzewicz, Z. W., Cambridge University Press, Cambridge, 104-108, 1995. 